\title{
Mise en place d'un élevage de Chironomus riparius Meigen (Diptera, Chironomidae) à l'aval d'une station d'épuration par
}

\section{lagunage}

\author{
V. Bouguenecl \\ N. Giani2
}

Mots clés : Chironomus riparius, système d'élevage, production, physico-chimie.

Un système d'élevage de Chironomus riparius Meigen a été mis en place sur le chenal d'amenée du rejet du $3^{e}$ bassin de la station d'épuration par lagunage de Baraqueville dans le département de l'Aveyron. Un suivi hebdomadaire des principaux facteurs physico-chimiques, sur une période d'un an, a permis de définir les caractéristiques et le fon-tionnement de ce système d'élevage. $51 \mathrm{~kg}$ de PF de larves ont été récoltés durant 183 jours d'exploitation (mai à octobre) soit $1,77 \mathrm{~kg}_{\text {de }} \mathrm{PS} \mathrm{ha}{ }^{-1} \mathrm{an}^{-1}$. La température, la teneur en $\mathrm{O}_{2}$ dissous et le taux de sédimentation sont les principaux facteurs susceptibles d'influencer la production.

Development of a rearing system for Chironomus riparius Meigen (Diptera, Chironomidae) below a waste-lagoon purification station

Keywords : Chironomus riparitus, rearing system, production, physico-chemical.

A rearing system for Chironomus riparius Meigen has been developed on the outlet channel of the third basin of a waste-lagoon purification station at Baraqueville in the department of Aveyron. A weekly survey of the principal physicochemical factors was made over a year and facilitated the description of the characteristics and functioning of the rearing system. $51 \mathrm{~kg}$ wet weight of larvae have been collected over 183 days of operation (May to October), equivalent to 1.77 $\mathrm{kg}$ dry weight ha-1 year ${ }^{-1}$. Temperature, dissolved oxygen concentration, and sedimentation rate are the principal factors affecting production.

\section{Introduction}

A ce jour, la majorité des expériences de valorisation des lagunes d'épuration ont porté sur les productions piscicole, planctonique ou macrophytique. Aucune valorisation intensive du benthos n'a été envisagée compte-tenu des difficultés de récolte ; seule une "cueillette » a parfois été pratiquée.

L'étude de la lagune d'épuration de Baraqueville (Aveyron) a montré que le dernier bassin abritait une importante population de Chironomus riparius Mei-

1. Institut Aquapure, Pradines, 12160 Baraqueville, France.

2. Laboratoire d'Hydrobiologie, URA 695 CNRS, Université Paul Sabatier, 118, route de Narbonne, F-31062 Toulouse Cedex, France. gen qui constituait l'une des composantes principales du benthos (Cauderlier 1985). La dégradation progressive du milieu de ce bassin a entraîné la disparition de cette espèce qui s'est alors installée dans le ruisseau récepteur, à l'aval du rejet. Cette évolution temporelle des lagunes d'épuration, encore non maîtrisable, nous a conduit à réaliser un élevage de Ch. riparius, à l'aval de la station d'épuration, dans un système artificiel facile à contrôler. Dans ce système, les principaux facteurs physiques ou chimiques, la production de $C h$. riparius et les quantités de matériaux importées ou exportées ont été étudiés pendant une année de façon à appréhender le fonctionnement du dispositif mis en place en vue de son optimisation. Les premiers résultats de cette étude sont présentés dans ce travail. 


\section{Matériel et méthodes}

La station d'épuration par lagunage de Baraqueville ( 2500 habitants) est située au S.O. du Massif Central, dans le département de l'Aveyron à $700 \mathrm{~m}$ d'altitude. Elle est constituée par une série de 3 bassins dont le dernier se déverse dans un petit ruisseau par un chenal long de $50 \mathrm{~m}$ environ (Fig. 1A et $1 \mathrm{~B}$ ). Ce chenal a été aménagé de façon à assurer un courant assez lent, une bonne oxygénation de l'eau, une accumulation de vase et une récolte facile des larves. Dix bacs en béton $(\mathrm{L}=4 \mathrm{~m}, 1=2 \mathrm{~m}, \mathrm{p}=$ $0,2 \mathrm{~m}$ ), se déversant en cascade les uns dans les autres (Fig. 1C), ont été construits sur ce chenal, entre le dernier bassin de la lagune et le ruisseau récepteur (Fig. 1B).

Un filet de $250 \mu \mathrm{m}$ de vide de maille, placé en permanence à l'exutoire de la lagune d'épuration
(Fig. 1C) capture les macto-invertébrés qui sont utilisés pour nourrir des poissons élevés expérimentalement sur le site. Des analyses physico-chimiques d'eau ont été réalisées chaque semaine, de novembre 1988 à octobre 1989, à l'entrée (E1) du système et à la sortie (Fig. 1C) de chacun des 10 bacs (S1 à S10). L'évolution journalière des principaux facteurs a été suivie sur des échantillons prélevés toutes les 3 heures au cours de cycles de 24 heures, en hiver (7-8/12/1988), au printemps (29-30/3/89), en été (11-12/07/89) et en automne (27-28/09/89). La température et l'oxygène dissous ont été mesurés à l'aide d'un appareil YSI 57, le $\mathrm{pH}$ avec un $\mathrm{pH}$-mètre Schott. Le débit a été évalué grâce à un caisson jaugé et à un micromoulinet Ott. Le temps de transit de l'eau dans le milieu a été mesuré par injection de fluorescéine.
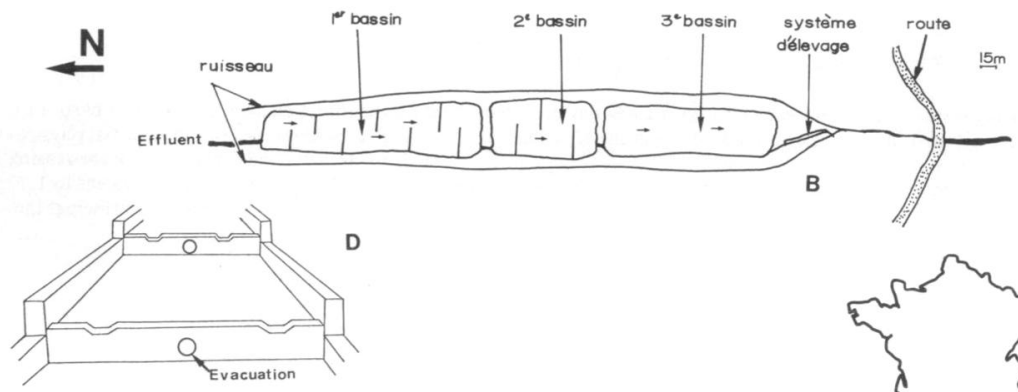

D

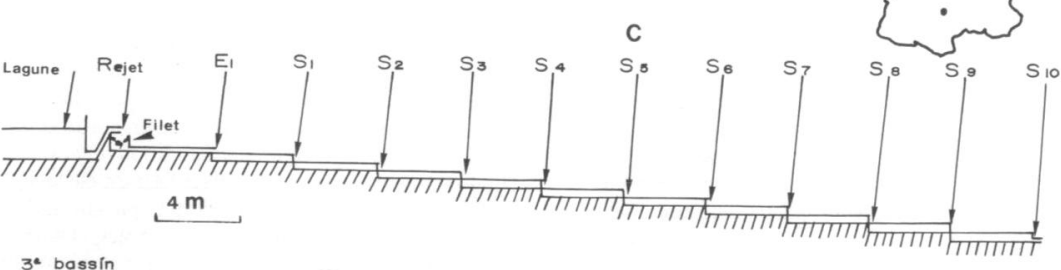

$3^{*}$ bassin

- Lagune - Système d'élevage

Fig. 1. Site d'étude : A : Localisation ; B : Plan de la lagune d'épuration et de son exutoire ; C : Plan du système d'élevage et localisation des points d'échantillonnage ; D : Plan de détail d'un bac.

Fig. 1. Study area : A : location, B : waste-lagoon plan and its outlet, C : breeding area plan and location of sampling points, D detailed plan of a pond. 
Des filtrations ont été réalisées à l'aide de filtres $\mathrm{GF} / \mathrm{C}$ et d'un filet de $70_{\mu} \mathrm{m}$ de vide de maille à l'entrée et à la sortie du système étudié. Le matériel récolté (matières en suspension : MES, invertébrés) a été égoutté et pesé au $1 / 100^{\circ}$ de gr., puis séché à l'étuve à $100^{\circ} \mathrm{C}$ pendant une nuit pour obtenir le poids $\mathrm{sec}$ au $1 / 10^{\circ}$ de $\mathrm{mg}$.

La quantité de matériaux qui sédimente a été mesurée dans des récipients en verre $(\varnothing=7,5 \mathrm{~cm}$, $\mathrm{h}=9 \mathrm{~cm}$ ) posés sur le fond des bacs.

Les larves de Chironomidés sont récoltées à l'aide d'un filet de $800{ }_{\mu} \mathrm{m}$ de vide de maille placé sur le tube d'évacuation inférieur des bacs (Fig. ID). Pendant la récolte, l'alimentation en eau du bac concerné est déviée. Le matériel ainsi récolté est lavé pour éliminer les particules fines puis égoutté ; il est ensuite étalé sur une grille métallique disposée audessus d'un bac rempli d'eau. Les Chironomidés migrent à travers la grille, dans l'eau du bac ; ils sont ensuite récupérés dans un filet, par vidange du bac, et pesés. Les Chironomidés ont été récoltés dans 5 des 10 bacs, en alternance, chaque semaine, soit bimensuellement pour un bac donné.

Le nombre de pontes a été estimé par comptage journalier sur des plaques de fibrociment à demiimmergées dans les bacs. Le nombre d'émergences imaginales journalières a été estimé dans 5 bacs à l'aide de pièges de type Mundie (1956) de $0,348 \mathrm{~m}^{2}$ de surface de base.

\section{Résultats}

\subsection{Caractéristiques physico-chimiques du milieu d'élevage}

\subsubsection{Débit et circulation de l'eau}

Le système mis en place augmente le temps de transit de l'eau dans le chenal; il varie de 1 h 30 à 9 heures entre le premier et le dernier bac $(40 \mathrm{~m})$; le temps de séjour de l'eau dans un bac est compris entre 2 et 53 minutes.

Le débit mesuré à l'entrée du système varie en cours d'année entre 0,06 et $3,61 \mathrm{~s}^{-1}$ avec une moyenne annuelle de $1,49 \pm 1,031 \mathrm{~s}^{-1}$. Le débit est, en général, plus faible à la sortie qu'à l'entrée (Fig. 2A) ; l'évaporation et le suintement au niveau des parois des bacs expliquent cette perte de charge. En été, l'évaporation, favorisée par l'augmentation de la durée du transit de l'eau dans le système, peut représenter jusqu'à $9 \%$ du débit de l'entrée.
Les variations journalières du débit (Fig. 3A) sont importantes et traduisent surtout les fluctuations de l'alimentation par les égouts et des précipitations. Ainsi, la décroissance continue du débit pendant le cycle de 24 heures de décembre (Fig. $3 \mathrm{~A}$ ) est due à l'élimination progressive des précipitations enregistrées les jours précédents et temporairement retenues dans les bassins de lagunage en amont.

\subsubsection{Température}

Au cours de l'année, la température de l'eau a varié entre $2,5^{\circ} \mathrm{C}$ en décembre et $23^{\circ} \mathrm{C}$ en juillet. Inférieure à $5^{\circ} \mathrm{C}$ pendant l'hiver, elle augmente brutalement en mai et reste supérieure à $15^{\circ} \mathrm{C}$ jusqu'à la fin août (Fig. 2B). Compte-tenu de la faible vitesse de circulation de l'eau, la température augmente de façon significative de l'entrée à la sortie du système : $1,5^{\circ} \mathrm{C}$ en moyenne et jusqu'à $6,5^{\circ} \mathrm{C}$ pendant l'été. Au contraire, en hiver, on note parfois une faible diminution de la température $\left(1,2^{\circ} \mathrm{C}\right.$ maximum) entre l'amont et l'aval.

Les écarts amont-aval au cours des 4 cycles de 24 heures (Fig. 3B) sont négatifs la nuit et positifs le jour ; leur amplitude, liée à la saison, est très faible en hiver et maximale en mars.

\subsection{3. $p H$}

Le $\mathrm{pH}$ a varié entre 7,4 et 9 . Il augmente assez régulièrement de $\mathrm{El}$ vers $\mathrm{S} 10$. Les écarts mesurés sur une distance de $40 \mathrm{~m}$ peuvent être importants et atteindre 1,3 unités (Fig. 2C) ; ils sont minimums en hiver et au début du printemps et maximums en été et à l'automne.

En hiver, le pH reste stable dans l'espace (E1 à S10) et dans le temps (Fig. 3C). Par contre, aux autres saisons, les variations journalières sont fortes ; le pH augmente brutalement le jour et décroît régulièrement la nuit (Fig. $3 \mathrm{C}$ ).

\subsubsection{Oxygène}

Les teneurs en oxygène s'échelonnent entre 4,8 et $17 \mathrm{ppm}^{-1}$. Elles augmentent en moyenne de $4,8 \pm$ $2,4 \mathrm{ppm}^{-1}$ (maximum : 7,7 ppm) de l'entrée à la sortie du système. Les taux de saturation augmentent de façon comparable entre El $(74,3 \pm 27,9 \%)$ et $S 10(125 \pm 41,1 \%)$. De novembre à mars, les teneurs en $\mathrm{O}_{2}$ dissous sont toujours inférieures au taux de saturation (de 41,6 à 97,8\%). Le reste de l'année, ce taux est toujours supérieur à $100 \%$ à partir de $\mathrm{S} 3$ et les plus fortes moyennes mensuelles sont enregistrées de mai à août (jusqu'à $189 \%$ ). 

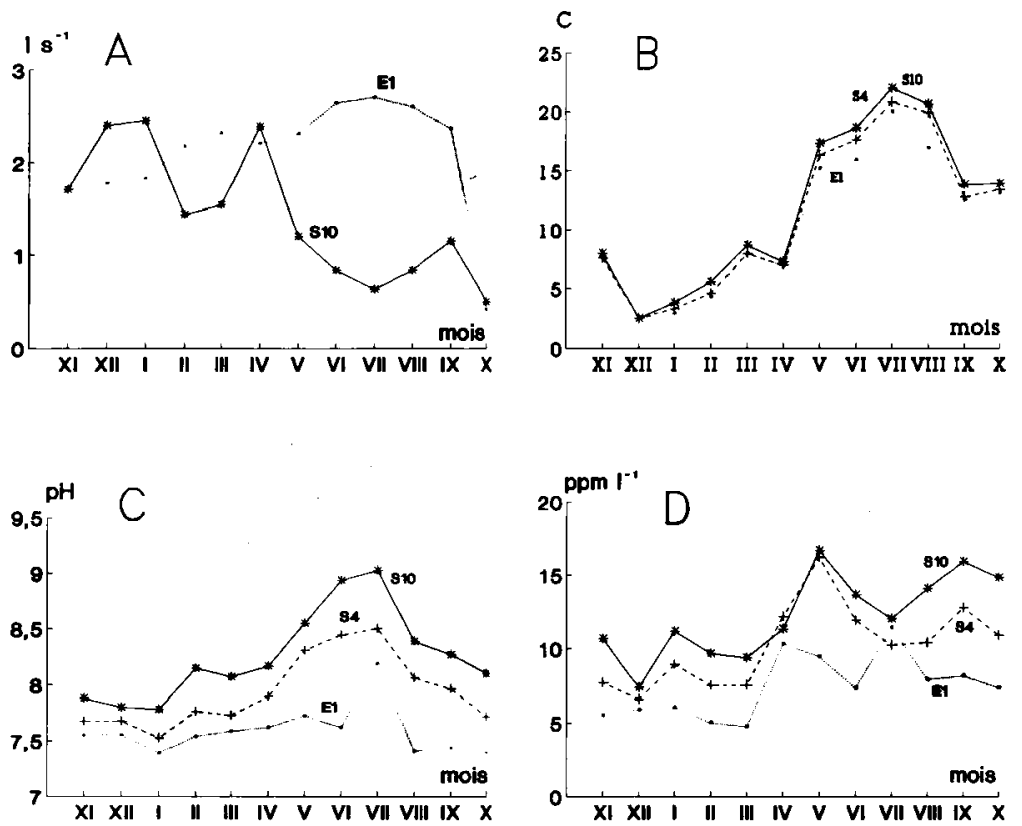

Fig. 2. A : Débits moyens annuels à l'entrée (E1) et à la sortie (S10) du système d'élevage. Evolution de la température moyenne (B), du $\mathrm{pH}(\mathrm{C})$ et des teneurs moyennes en $\mathrm{O}_{2}$ (D) au cours de l'année en E1, S4 et S10.

Fig. 2. $A$ : annual mean water flow in $E 1$ and $S 10$ of the breeding area ; $B$ : monthly mean temperature in $E 1, S 4$ and $S 10 ; C:$ annual pH evolution in E1, S4 and S10; D : annual mean $\mathrm{O}_{2}$ evolution in E1, S4 and $\$ 10$.

Au sein d'un même bac, les variations annuelles

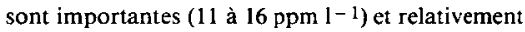
identiques pour tous les bacs (Fig. 2D) ; les variations journalières (Fig. 3D) sont similaires à celles $\mathrm{du} \mathrm{pH}$.

\subsubsection{Sédimentation}

Le taux de sédimentation diminue entre E1 et S5 et augmente ensuite jusqu'à S10 (Fig. 4). Les quantités moyennes de sédiments déposés varient fortement au sein d'un même bac au cours de l'année : par exemple de 26 à $213 \mathrm{~g}$ poids sec bac $^{-1}$ jour $^{-1}$ pour le bac $\mathrm{S} 7$. Ces quantités sont maximales en août et minimales en hiver (Tableau 1). La quantité annuelle moyenne de sédiments déposés est de 325 $\pm 32 \mathrm{~kg}$ pour l'ensemble du système avec 55 à $84 \%$ de matière organique (Fig. 4).

\subsubsection{Matières en suspension : MES}

Les quantités moyennes mensuelles de MES en E1 et $\mathrm{S} 10$ sont comparables et voisines de $68 \mathrm{~g} \mathrm{~m}^{-3}$; elles varient au cours de l'année de 5,2 à $200 \mathrm{~g} \mathrm{~m}^{-3}$ avec des maximums en octobre, février et mars et des minimums durant les mois d'été. 

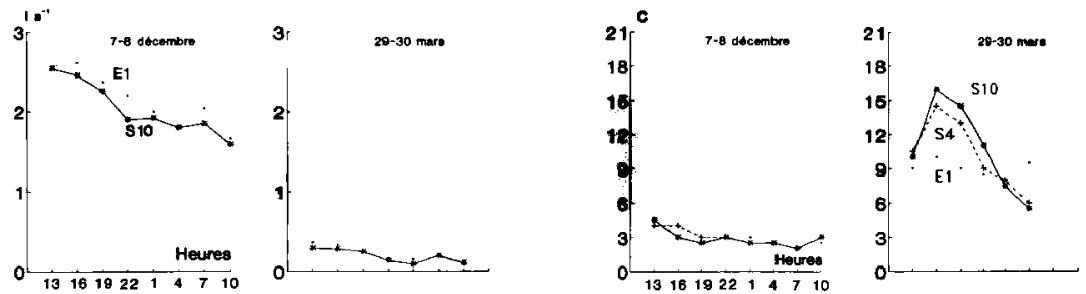

\section{A}
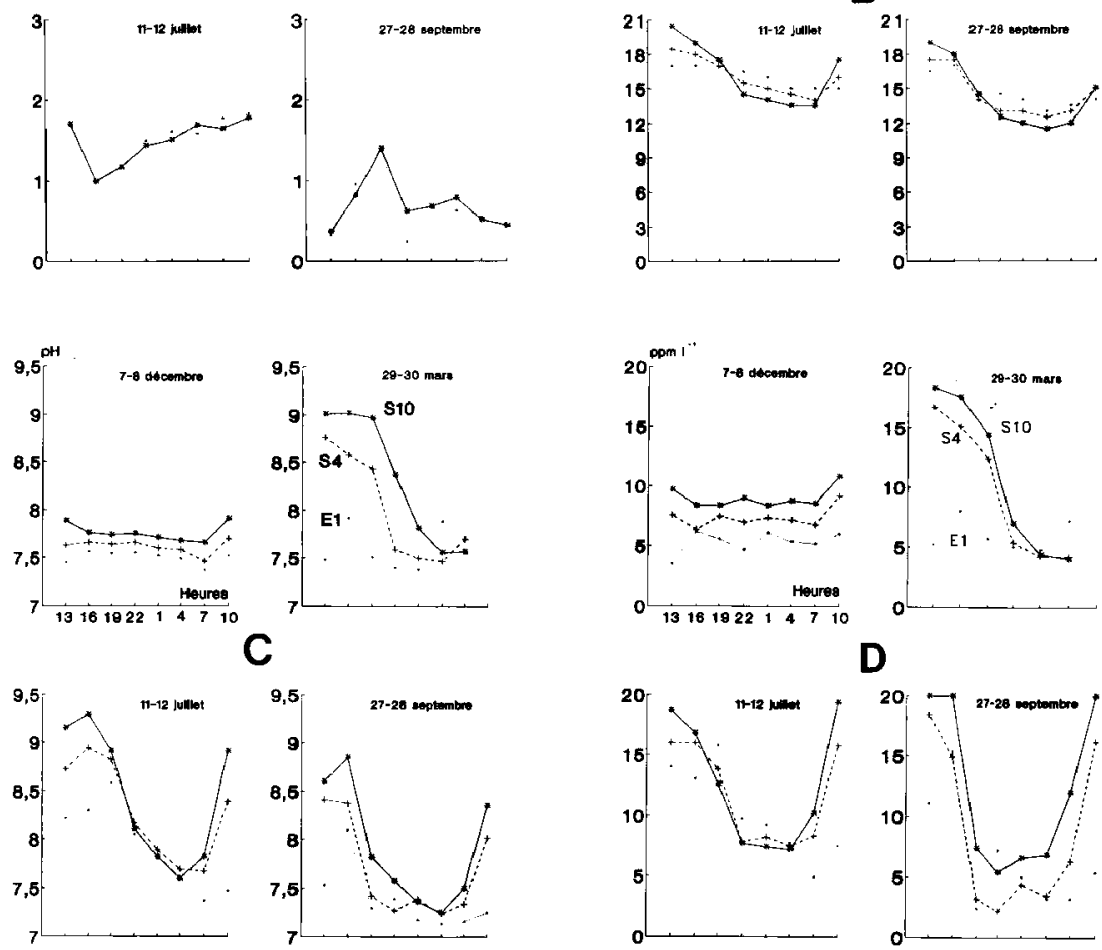

Fig. 3. A : Variation du débit au cours des cycles de $24 \mathrm{H}$ à l'entrée (E1) et à la sortie (S10) du système d'élevage. Evolution de la température (B), du pH (C) et des teneurs en $\mathrm{O}_{2}$ (D) au cours des 4 cycles de 24 heures en E1, S4 et S10.

Fig. 3. A : variation in the flow regime $\left(1 \mathrm{~s}^{-1}\right)$ during the $24 \mathrm{H}$ survey in $\mathrm{E} 1$ and $\mathrm{S} 10$; $\mathrm{B}$ : evolution of the temperature during the daily cycles in E1, S4 and $\mathrm{S10} ; \mathrm{C}$ : daily $\mathrm{pH}$ evolution in E1, S4 and S10; D : daily $\mathrm{O}_{2}$ evolution in E1, S4 and S10. 
Tableau 1. Variation annuelle de la quantité moyenne de sédiments déposés dans 5 bacs d'élevage (en $\mathrm{g} \mathrm{de} \mathrm{PS} \mathrm{bac}^{-1}$ jour ${ }^{-1}$ ). Table 1 . Monthly means of the weight of sediment deposited each day in the various ponds (in dry weight, $\mathrm{g}^{\text {pond }}{ }^{-1}$ day ${ }^{-1}$ ).

\begin{tabular}{ccrrrrrr}
\hline Mois & I & II & III & V & VI & VIII & IX \\
\hline Bac 1 & 57,75 & 64,96 & 51,71 & 171,85 & 124,85 & 157,71 & 23,97 \\
Bac 3 & 49,23 & 51,12 & 38,49 & 117,73 & 73,48 & 188,93 & 67,78 \\
Bac 5 & 53,24 & 63,84 & 57,38 & 109,69 & 54,21 & 139,33 & 82,27 \\
Bac 7 & 48,05 & 65,68 & 53,62 & 61,88 & 132,38 & 213,73 & 26,48 \\
Bac 10 & 39,52 & 67,52 & 47,94 & 63,47 & 134,24 & 283,77 & 77,71 \\
\hline
\end{tabular}

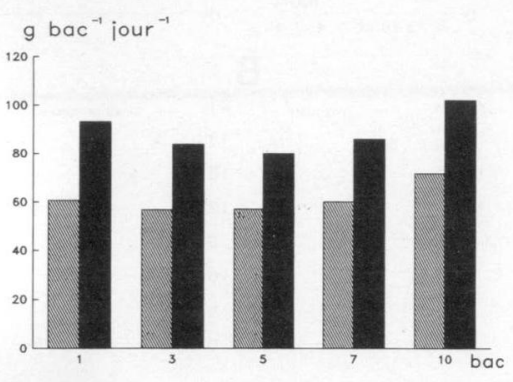

Fig. 4. Evolution spatiale de la quantité totale de sédiments déposés (noir) et de leur teneur en matière organique (hâchuré) dans le système d'élevage.

Fig. 4. Spatial evolution of the total quantity of sediments deposited and their organic content $(\%)$ in the breeding area (organic matter MO : hatched, total matter: filled).

En cours de journée, les quantités de MES ne diffèrent pas significativement d'un bac à l'autre. A l'exception de décembre, les variations journalières au sein d'un bac sont très marquées avec des minima nocturnes et des maxima diurnes (Fig. 5A) ; c'est en juillet que les écarts sont les plus prononcés. La teneur en matière organique des MES est toujours élevée et comprise entre 90 et $100 \%$.

\subsection{Récolte des invertébrés}

Entre 126 et $303 \mathrm{~kg}$ PF d'invertébrés, de taille comprise entre 70 et $250 \mu \mathrm{m}$, entrent par an dans le système. Les quantités moyennes mensuelles d'invertébrés exportées en S10 sont supérieures d'environ $26,8 \%$ à celles importées en $\mathrm{E} 1$, sauf en novembre et décembre (Fig. 6). Les quantités de matériel qui transitent dans le système (Tableau 2) sont très variables.
Les flux journaliers d'invertébrés qui entrent ou sortent du système présentent des maxima d'abondance diurnes en septembre et nocturnes en mars et juillet en $\mathrm{E} 1$ (Fig. 5B). Ces variations journalières sont plus marquées en E1 qu'en S10 (Fig. 5B) avec un décalage des maxima de 6 à $12 \mathrm{~h}$, consécutif au temps de transit de l'eau de El à S10.

Parmi ces invertébrés, peu de Chironomus riparius $\mathrm{Mg}$. ont été récoltés dans les filtrations de décembre, mars et septembre en El comme en S10. Dans l'année, $54 \pm 20 \mathrm{~g}$ PS de $C h$. riparius entrent dans le système et $115 \pm 45 \mathrm{~g}$ PS en sortent. En juillet, les 4 stades larvaires de $C h$. riparius sont présents à l'entrée et à la sortie mais les stades IV dominent largement : $53,5 \%$ en S10 (Fig. 7A); les nymphes ne représentent que $1,5 \%$ du matériel exporté.

Annuellement, $615 \pm 128 \mathrm{mg}$ PS d'exuvies nymphales $(n=8412)$ ont été récoltées à l'entrée et seulement $109 \pm 23 \mathrm{mg}$ PS $(n=1488)$ à la sortie. Les exuvies sont essentiellement présentes de juin à août, période maxima des émergences imaginales. La faible quantité d'exuvies collectées (Fig. 7B) résulte de l'emplacement des filets sur les tuyaux d'évacuation situés sous la surface de l'eau (Fig. 1D), les privant de la dérive de surface.

Les autres invertébrés capturés dans les filtrations sont en majorité des Rotifères et des Chaoboridae à l'entrée. A la sortie, apparaissent des Culicidae ainsi que 8 espèces de Chironomidae : Ablabesmyia monilis (L.), Cricotopus ornatus (Mg.), Metriocnemus hygropetricus K., Paraphaenocladius penerasus Edw., Glyptotendipes barbipes (Staeg.), Polypedilum convictum (Walk.), Polypedilum nubeculosum (Mg.) et Micropsectra atrofasciata (K.).

En mars, période où les Chironomidés sont absents des filtrations, nous avons relevé la composition suivante : 

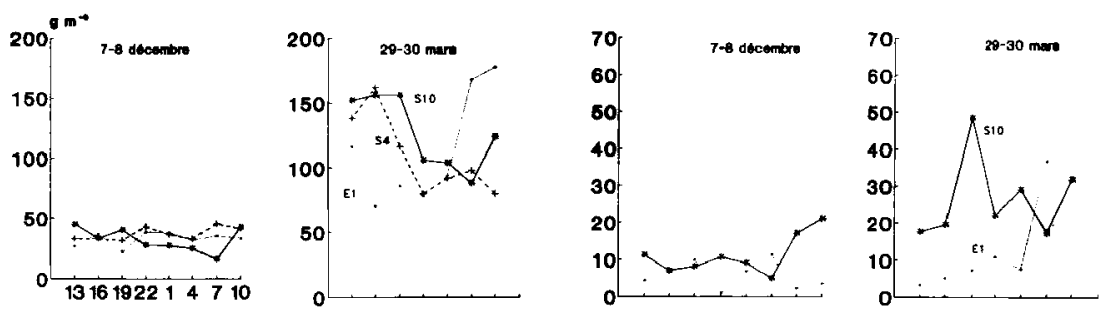

A

B
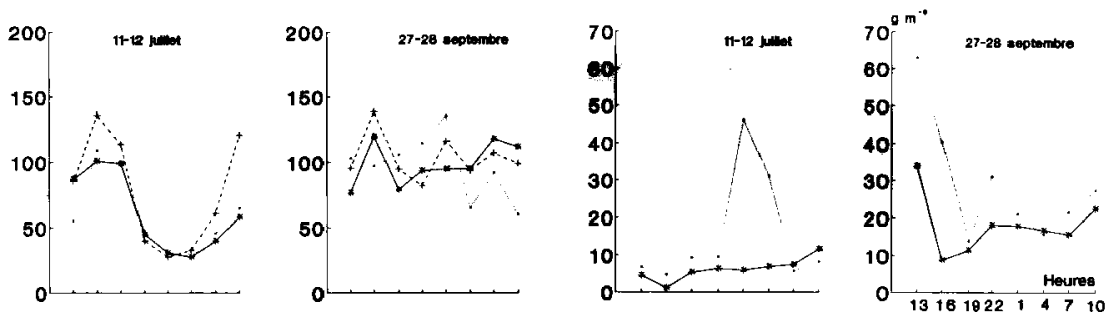

Fig. 5. A : Variations journalières des MES déposées en E1, S4 et S10; B : Quantités d'invertébrés ( $\left.\mathrm{g} \mathrm{m}^{-3}\right)$ à l'entrée $(\mathrm{E} 1)$ et à la sortie (S10) du système d'élevage au cours des 4 cycles de 24 heures.

Fig. 5. A : daily variation of the MES in El, S4 and $S 10 ; B$ : daily variation of invertebrate biomass $\left(g \mathrm{~m}^{-3}\right)$ in $E I$ and $S 10$.

Tableau 2. Quartités moyennes ( $\mathrm{g} \mathrm{m}^{-3}$ ) annuelles et journalières d'invertébrès (PF : poids frais, PS : poids sec) qui entrent et sortent du système d'élevage.

Table 2. Mean annual and daily quantities $\left(\mathrm{g} \mathrm{m}^{-3}\right)$ of invertebrates (PF : fresh weight, PS : dry weight) entering and exported from the breeding system.

\begin{tabular}{|c|c|c|c|c|}
\hline & \multicolumn{2}{|c|}{ Entrée (E1) } & \multicolumn{2}{|c|}{ Sortie (S10) } \\
\hline & $\mathrm{g} / \mathrm{PF} \mathrm{m}^{-3}$ & $\mathrm{~g} / \mathrm{PS} \mathrm{m}^{-3}$ & $\mathrm{~g} / \mathrm{PF} \mathrm{m}^{-3}$ & $\mathrm{~g} / \mathrm{PS} \mathrm{m}^{-3}$ \\
\hline$\overline{\mathrm{x}}$ annuelle & $16,17 \pm 46,07$ & $0,85 \pm 2,01$ & $16,30 \pm 57,60$ & $1,05 \pm 2,01$ \\
\hline 7-8 décembre & $5,72 \pm 3,61$ & $0,46 \pm 0,24$ & $11,17 \pm 5,32$ & $0,64 \pm 0,28$ \\
\hline $29-30$ mars & $14,84 \pm 13,85$ & $0,97 \pm 1,14$ & $26,57 \pm 11,13$ & $1,77 \pm 0,82$ \\
\hline 11-12 juillet & $15,18=15,16$ & $1,53 \pm 1,73$ & $6,19 \pm 2,93$ & $0,52 \pm 0,34$ \\
\hline 27.28 septembre & $29,24 \pm 16,12$ & $1,15 \pm 0,69$ & $18,19 \pm 7,63$ & $0,39 \pm 0,28$ \\
\hline
\end{tabular}




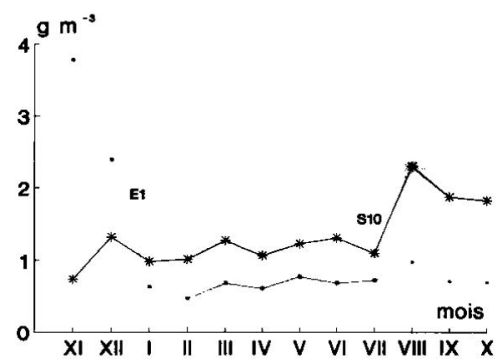

Fig. 6. Biomasse d'invertébrés $\left(\mathrm{g} \mathrm{m}^{-3}\right)$ a l'entrée (E1) et à la sortie (S10) du système d'élevage au cours de l'année.

Fig. 6. Annual biomass of invertebrates $\left(\mathrm{g} \mathrm{m}^{-3}\right)$ in $\mathrm{E} 1$ and $\mathrm{S} 10$.

\begin{tabular}{lrrrr} 
& \multicolumn{1}{c}{ Entrée } & \multicolumn{2}{c}{ Sortie } \\
Rotifères & 78,47 & $\%$ & 99,70 & $\%$ \\
Naididae & 0,28 & $\%$ & 0,002 & $\%$ \\
Chaoborus & 21,37 & $\%$ & 0,04 & $\%$ \\
Simulies & 0,14 & $\%$ & & \\
Copépodes & 0,14 & $\%$ & 0,25 & $\%$
\end{tabular}

\subsection{Production de Chironomus riparius}

La période où la production de $C h$. riparius est suffisante pour être exploitée dure 4 mois, du 15 mai au 15 septembre. $51 \mathrm{~kg}$ PF de larves ont été récoltés en 183 jours d'exploitation soit 7,12 kg PS (PF/PS $=7,16 \pm 0,96$ ). Il existe deux pics de production, la première quinzaine de juin et la première quinzaine d'août avec une diminution au milieu de l'été (Fig. 8A). Les plus fortes productions sont concentrées en juin $(30,2 \%)$ et en août $(29,2 \%)$. La production dans les 5 derniers bacs $(28,2 \mathrm{~kg})$ est légèrement supérieure à celle des 5 bacs amont $(22,6 \mathrm{~kg})$ (Fig. 8B). La production de chaque bac a varié entre $4 \mathrm{~kg}$ (bac 4) et $6,7 \mathrm{~kg}$ (bac 6) avec des productions journalières de 2,7 à $4,6 \mathrm{~g} \mathrm{~m}^{-2} \mathrm{j}^{-1}$. La production des larves dans le système d'élevage doit être corrigée en fonction de pertes diverses:

- pertes de $0,46 \%$ lors de la récolte (estimées à l'aide d'un filet placé en sortie du système lors de la récolte) ;

- pertes lors de l'extraction sur grille, de l'ordre de 0,15 à $15,27 \%$, soit en moyenne $3,93 \pm$ $5,62 \%$ de la récolte.

Après réajustement, ce sont $7,44 \mathrm{~kg}$ PS de larves qui ont été extraites du système pendant les 4 mois de production.
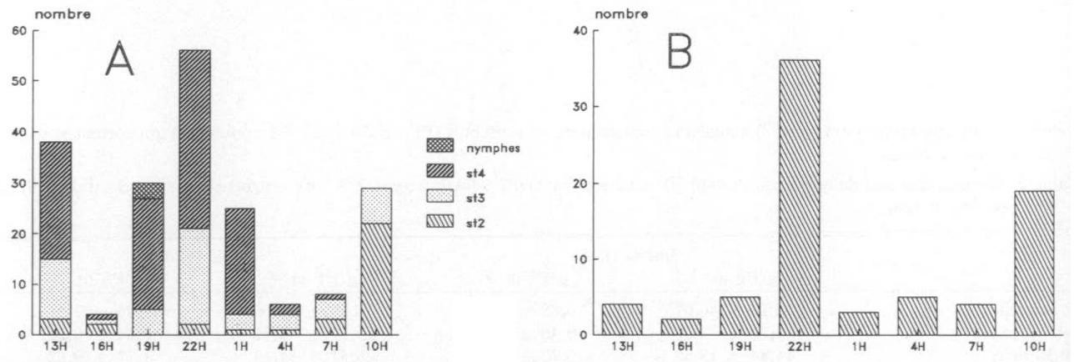

Fig. 7. A : Nombre de larves aux différents stades et de nymphes récoltées par filtration en 24 heures le 11 juillet ; B : Variation du nombre d'exuvies nymphales récoltées par filtration en juillet.

Fig. 7. A : number of larvae in the four instars and number of pupae in a July drift sample ; $B$ : daily number of pupal exuviae in a July drift sample. 

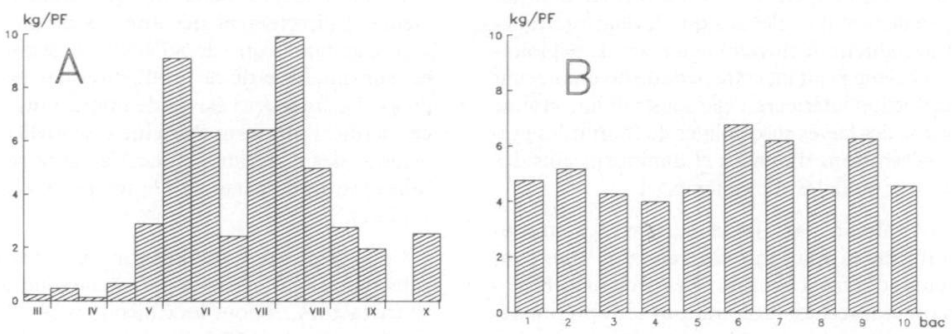

Fig. 8. A : Poids (kg PF) de Chironomus riparius récoltés dans le système d'élevage, chaque quinzaine, de mars à octobre ; $B$ : Production annuelle de Chironomus riparius ( $\mathrm{kg}$ de $\mathrm{PF}$ ) dans les 10 bacs du système d'èlevage.

Fig. 8. A : biomass of Chironomus riparius harvested every fortnight ; B : annual production of Chironomus riparius in the various ponds of the breeding area.

38548 pontes ont été déposées dans le système dont $45 \%$ entre mai et juin : leur poids atteint 12,4 $\pm 0,3 \mathrm{~g}$ PS.

Entre juin et octobre, 19213 imagos or ot 27624 imagos $Q Q$ ont émergé. Compte-tenu des poids individuels moyens (Tableau 3 ), ce sont 128,28 $\pm 24,47 \mathrm{~g}$ PF ou $42,44 \pm 20,76 \mathrm{~g}$ PS d'imagos qui sont exportés hors du système.

\section{Discussion}

Les données recueillies au cours d'une année d'étude sur le système d'élevage ont permis d'appréhender son fonctionnement et notamment de préciser l'influence des principaux facteurs physicochimiques. Ainsi, afin d'accélérer le développement larvaire, le système d'élevage devait permettre d'augmenter la température de l'eau dès sa sortie de la lagune. Ainsi, l'écoulement quasi-laminaire (hauteur de la lame d'eau : 15 à $20 \mathrm{~cm}$ ) et l'allongement du temps de circulation entre le point de rejet et le ruisseau récepteur permettent un gain de quelques degrés de la température. La lagune joue un rôle important de réservoir thermique qui amortit les variations saisonnières, tandis que le système d'élevage reste plus sensible aux variations thermiques, dues aux déperditions (nocturnes, par temps couvert...) ou aux réchauffements. De ce fait, l'entróe du système est plus stable thermiquement que les bacs aval plus sensibles aux variations saisonnières ou journalières. Ce sont ces bacs situés à l'aval qui atteignent les températures les plus élevées. Au printemps et en automne, les amplitudes thermiques journalières peuvent atteindre $10^{\circ}$ à $11^{\circ} \mathrm{C}$ entre l'amont et l'aval ou au sein d'un même bac. Au contraire, l'été et surtout l'hiver sont des saisons de plus grande stabilité

Tableau 3. Poids moyen (PF et PS en mg) des divers stades larvaires (1, 2, 3 et 4), nymphes ( $N$ ), adultes (femelle : adf, mâle : adm), exuvies (femelle : exf, mâle : exm) et des pontes (p) de Chironomus riparius (n : nombre d'individus, sd : écart-type).

Table 3. Mean weight (PF and PS $\mathrm{mg}$ ) of the larval instars (1, 2,3 and 4), pupae (N), adults (female adf, male adm), pupal exuviae (female exf, male exm) and egg masses (P) of Chironomus riparius ( $\mathbf{n}$ : number of individuals, sd : standard deviation).

\begin{tabular}{|c|c|c|c|c|c|c|c|c|c|c|}
\hline Stades & 1 & 2 & 3 & 4 & $\mathbf{N}$ & adf & adm & exf & exm & $\mathbf{p}$ \\
\hline PF & 0,0161 & 0,182 & 0,513 & 4,05 & 3,97 & 1,769 & 1,307 & 2,38 & 2,274 & 26,5 \\
\hline PS & 0,00538 & 0,0231 & 0,052 & 0,78 & 1,49 & 0,587 & 0,43 & 0,0724 & 0,0738 & 0,3227 \\
\hline$s d$ & 0,0007 & 0,008 & 0,009 & 0,35 & 0,11 & 0,038 & 0,0077 & 0,0177 & 0,0127 & 0,0095 \\
\hline
\end{tabular}


thermique. La température est un facteur limitant de la production de l'élevage qui devient pratiquement improductif de novembre à mars. L'exploitation du système pendant cette période hivernale nuit à sa production ultérieure : elle soustrait une grande proportion des larves susceptibles de fournir les premières générations d'imagos et diminuent ainsi les potentialités initiales du système.

De novembre à mars, les teneurs en $\mathrm{O}_{2}$ sont toujours inférieures à la saturation alors qu'elles sont supérieures d'avril à octobre. Les processus biologiques (photosynthèse, respiration) sont prépondérants pour déterminer ces teneurs en $\mathrm{O}_{2}$. L'aération physique de l'eau, engendrée par la configuration du système d'élevage, est cependant indispensable entre le rejet de la lagune et le premier bac. Au-delà, elle ne se justifie que la nuit où elle permet de maintenir la teneur en $\mathrm{O}_{2}$ à un niveau compatible à la survie des larves.
De E1 à S5, les matériaux qui sédimentent proviennent directement des apports du $3^{e}$ bassin de lagunage tandis que de S6 à S10, la sédimentation est surtout issue de la production des bacs précédents. Les taux très élevés de matière organique de ces derniers prouvent l'origine essentiellement biogénique des matériaux et leur faible taux de minéralisation traduit leur faible temps de séjour dans les bacs.

Les MES, après sédimentation, constituent le substrat de l'élevage et la principale source de nourriture (algues...) pour les Chironomidés. Le budget entrée-sortie des MES est globalement équilibré et une grande partie du matériel qui sédimente ou est exporté est produite à l'intérieur du système (Fig. 9). La production de larves ne représente que 3,1\% de la quantité de matière organique qui sédimente. Les conditions trophiques ne semblent donc pas un facteur limitant de la productivité de l'élevage.

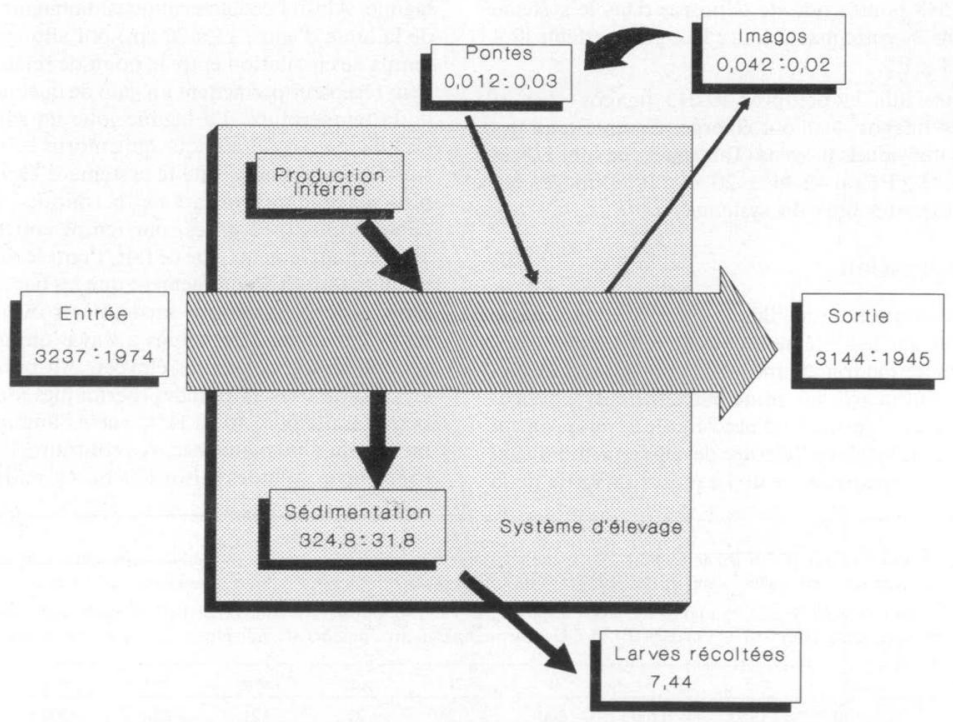

Fig. 9. Diagramme du fonctionnement du système d'élevage (kg de PS an-1).

Fig. 9. Functioning diagram of the breeding area (kg PS year ${ }^{-1}$ ). 
Les données de la littérature concernant les élevages intensifs de Chironomidés sont peu nombreuses. Les Chironomidés sont élevés en hors-sol (Konstantinov 1958), dans des bacs artificiels enrichis de fumier de volaille ou de farine de poisson (Yashouv 1970) ou dans des milieux naturels enrichis : dans des champs endigués à la manière des rizières et recevant un épandage de fumier de volaille (Shaw \& Mark 1980). Afin de rendre les résultats de ces différents élevages comparables, les valeurs de la production ont été converties en PS (PF/PS $=7,16 \pm$ $0,96)$ et calculées pour une même surface (ha) et une même période (année). La production obtenue dans le système d'élevage est supérieure à celle de l'ensemble des Chironomidés (Tableau 4) du $3^{\mathrm{e}}$ bassin de la lagune (Cauderlier 1985). Par contre, si nos résultats se rapprochent de ceux de Shaw \& Mark (1980), ils sont très inférieurs à ceux obtenus par Konstantinov (1958) en élevage hors-sol et par Yashouv (1970) en Israël, où la production n'est pas interrompue pendant l'hiver, la température étant toujours supérieure à $8^{\circ} \mathrm{C}$.

Tableau 4. Comparaison des productions (en $\mathrm{kg}$ de PS ha-1 $\mathrm{an}^{-1}$ ) des Chironomus obtenues à Baraqueville avec celles de la littérature.

Table 4. Comparison of production ( $\mathrm{kg} \mathrm{PS} \mathrm{ha}^{-1} \mathrm{year}^{-1}$ ) of Chironomus obtained in Baraqueville with values in the literature.

\begin{tabular}{|c|c|}
\hline Références & $\begin{array}{c}\text { Production } \\
\text { kg de PS ha } \\
\text {.1 } \text { an }^{-1}\end{array}$ \\
\hline Shaw \& Mark (1980) & $2-2,11$ \\
\hline Yashouv (1970) & $18,1-27,2$ \\
\hline Konstantinov (1958) & $4,8-8,1$ \\
\hline Jana \& Pal (1990) & $0,09-0,16$ \\
\hline Cauderlier (1985) & 0,199 \\
\hline Baraqueville & 1,77 \\
\hline
\end{tabular}

Nos résultats font état de la production récoltée et non de la production globale (production secondaire) telle qu'elle est calculée généralement en écologie. Toutefois, à titre de comparaison, nous avons relevé quelques chiffres relatifs à la production en milieu naturel :
- 9,5-15,3 $\mathrm{kg} \mathrm{ha}^{-1} \mathrm{an}^{-1}$ pour Chironomus plumosus L. dans un lac eutrophe du sud-ouest de l'Allemagne (Frank 1982).

$-6,29 \mathrm{~kg} \mathrm{ha}^{-1} \mathrm{an}^{-1}$ pour Chironomus commutatus Str. dans un lac de haute montagne des Pyrénées (Laville 1975).

L'assèchement temporaire des bacs, au moment de la récolte des larves, est certainement responsable d'une forte réduction de la production consécutive à la perte des premiers stades larvaires et à la destruction de pontes. Une technique de récolte qui, sans assécher les bacs, permettrait de conserver les jeunes stades et les pontes dans le milieu en ne prélevant que les stades IV est actuellement en cours d'étude. Elle permettrait d'augmenter la fréquence des récoltes sans porter préjudice à l'élevage.

\section{Remerciements}

Nous remercions H. Laville, du Laboratoire d'Hydrobiologie, qui a identifié les diverses espèces de Chironomidae rencontrées au cours de cette étude.

\section{Travaux cités}

Cauderlier E. 1985. - Etude de la physico-chimie et de la production planctonique d'une lagune d'épuration (lagune de Baraqueville, $700 \mathrm{~m}$ d'altitude) en vue de sa valorisation piscicole. Thèse de $3^{e}$ cycle, Toulouse IIl : $195 \mathrm{p}$.

Frank C. 1982. - Ecology, production and anaerobic metabolism of Chironomus plumosus $\mathbf{L}$. larvae in a shallow lake. I Ecology and production. Arch. Hydrobiol., 94 (4) : 460-491.

Jana B.B. \& Pal G.P. 1990. - Production of chironomid larvae in culturing media of various organic wastes. Limnologica. (Berl.), 21 (1) : 281-285.

Konstantinov A.S. 1958. - Biology of the Chironomidae and their cultivation. (VNIORCH), Saratov, USSR : 362 p (en russe).

Laville H. 1975. - Production d'un chironomide semivoltin (Chironomus commutatus Str.) dans le lac de Port-Bielh (Pyrénées Centrales). Annis Limnol, 2 (1):67-77.

Mundie J.H. 1956. - Emergence traps for aquatic insects. Mit. int. Ver, Limnol., $7: 13 \mathrm{p}$.

Shaw P.C. \& Mark K.K. 1980. - Chironomid farming. A means recycling farm manure and potentially reducing water pollution in Hong Kong. Aquaculture, 21 : 155-163.

Yashouv A. 1970. - Propagation of chironomid larva as food for fish fry. Bamiidgeh : 101-105. 